\title{
Décontamination nucléaire par laser UV
}

\author{
Ph. Delaporte, M. Gastaud, W. Marine ${ }^{1}$, M. Sentis, O. Uteza, P. Thouvenot ${ }^{2}$, \\ J.L. Alcaraz ${ }^{2}$, J.M. Le Samedy ${ }^{2}$ et D. Blin $^{3}$
}

Laboratoire Lasers, Plasmas et Procédés Photoniques, LP3, FRE 2165 du CNRS, Université de la Méditerranée, Pôle Scientifique de Luminy, 163 avenue de Luminy, Case 917, 13288 Marseille cedex 9, France

${ }^{1}$ Groupe de Physique des États Condensés (GPEC), UMR 6631 du CNRS, Université de la Méditerranée, Pôle Scientifique de Luminy, 163 avenue de Luminy, Case 901 , 13288 Marseille cedex 9, France

${ }^{2}$ Commissariat à l'Énergie Atomique, Centre d'Études Nucléaires de Cadarache, DEN/DED/SEP, 13108 Saint-Paul-lez-Durance, France

${ }^{3}$ ONECTRA, Traverse Pomègues, 13008 Marseille, France

\begin{abstract}
Résumé : Le développement et l'utilisation de procédés propres pour le nettoyage ou la préparation de surfaces est l'une des priorités du milieu industriel. Cet intérêt est d'autant plus grand dans le domaine du nucléaire pour lequel la réduction des déchets est un axe de recherche important. Un dispositif de décontamination nucléaire par laser UV impulsionnel a été développé et testé. Il est composé d'un laser à excimères de $1 \mathrm{~kW}$, d'un faisceau de fibres optiques et d'un dispositif de récupération des particules. Les essais réalisés en milieu actif ont démontré sa capacité à nettoyer des surfaces métalliques polluées par différents radioéléments avec des facteurs de décontamination généralement supérieurs à 10. Ce dispositif permet de décontaminer de grandes surfaces de géométrie simple en réduisant fortement la génération de déchets secondaires. Il est, à ce jour et dans ces conditions d'utilisations, le procédé de décontamination par voie sèche le plus efficace.
\end{abstract}

\section{INTRODUCTION}

La décontamination de surface est un problème critique dans le milieu nucléaire. Les caractéristiques (matériau, taille, forme, ...) des objets à décontaminer sont très diverses. Dans le cas des matériaux métalliques, les radioéléments sont généralement piégés dans la couche d'oxyde qui se forme à la surface. Les techniques actuellement utilisées pour décontaminer de grandes surfaces sont soit des procédés chimiques, soit des procédés basés sur la projection de particules. Cependant, ce sont des procédés par voie humide qui génèrent une quantité importante de déchets secondaires qu'il est nécessaire de stocker. Le développement d'un procédé sec de décontamination qui limiterait cette production de déchets est donc particulièrement intéressante.

Plusieurs applications des lasers impulsionnels (YAG et excimères) ont déjà été développées dans le domaine du traitement ou du nettoyage de surface [1-2]. Pour démontrer la possibilité d'utiliser ce type de source laser pour l'enlèvement de couches d'oxydes métalliques contaminées, nous avons tout d'abord développé un prototype de décontamination par laser [3]. Puis, des essais de validation ont été réalisés au CEA Cadarache avec des échantillons provenant d'installations nucléaires.

\section{PROTOTYPE DE NETTOYAGE PAR LASER}

Lorsqu'une couche d'oxyde est irradiée par un faisceau laser, elle absorbe l'énergie des photons incidents et cela peut conduire à l'ablation d'une fine épaisseur de cette couche. Pour les matériaux métalliques que nous avons étudiés, l'absorptivité est inversement proportionnelle à la longueur d'onde et l'utilisation de courtes longueurs d'onde permet de générer un processus d'ablation plus efficace [4-5]. De plus, l'irradiation avec des durées d'impulsions courtes limite les effets thermiques en profondeur, et réduit les modifications des propriétés du matériau. Un laser à excimères a donc été choisi pour développer le prototype de décontamination. Afin de réduire les pertes d'énergie induites lors du transport du faisceau par fibres optiques par la formation de centres colorés de types E' dans le cœur des fibres en silice, nous avons utilisé un laser XeCl (308nm), plutôt que des lasers $\mathrm{KrF}(248 \mathrm{~nm})$ ou ArF (193nm). Lors du 
développement de ce prototype, deux sources laser ont été utilisées : un Lambda Physics EMG 203 MSC $(25 \mathrm{~ns}, 400 \mathrm{~mJ}, 200 \mathrm{~Hz})$ et le CILAS UV $635(70 \mathrm{~ns}, 3 \mathrm{~J}, 400 \mathrm{~Hz})$.

Le plasma d'ablation qui se propage perpendiculairement à la surface contient les particules radioactives, et il est nécessaire de les collecter pour éviter leur redéposition. Une buse d'aspiration, placée à quelques millimètres de la surface, et reliée à un système de pompage via un filtre permet la récupération des produits d'ablation. Un système optique, intégré dans la buse d'aspiration et protégé par un flux de gaz neutre, réalise la remise en forme du faisceau laser à la sortie des fibres optiques.

Le faisceau de fibres optiques réalisé pour transporter le faisceau d'un laser $\mathrm{XeCl}$ d'une puissance moyenne de $300 \mathrm{~W}(1 \mathrm{~J}, 300 \mathrm{~Hz})$, est constitué de 90 fibres en silice de 5 mètres de long. Un rendement de transmission ( $\mathrm{E}_{\text {cible }} / \mathrm{E}_{\text {laser }}$ ) de $50 \%$ a été obtenu [6].

Pour permettre une automatisation complète du procédé, la qualité du nettoyage doit être contrôlée en temps réel. La présence de radioéléments dans le plasma d'ablation est déterminée par analyse spectroscopique. La détection dans le plasma de l'élément considéré (par exemple un pic à $852 \mathrm{~nm}$ pour le césium) permet d'en déduire la présence de cet élément sur la zone traitée. Lorsque la zone est totalement décontaminée, l'émission disparaît et le faisceau peut être déplacé vers une autre zone. Une seconde technique, basée sur la détection et l'analyse du champ électrique créé par le plasma, a également été développée [7].

\section{DISPOSITIF EXPERIMENTAL}

Le laser Lambda Physics EMG 203 MSC a été utilisé pour effectuer les validations expérimentales de décontamination par laser réalisée au CEA Cadarache. Les échantillons contaminés, la buse d'aspiration et le filtre étaient placés dans une boîte à gants. Des tables motorisées permettaient le déplacement des pièces à traiter devant le faisceau. Le laser et le dispositif de mise en forme du faisceau étaient situés à l'extérieur de la boîte à gants. Un passage étanche fermé par un hublot en silice UV permettait au faisceau d'atteindre la surface contaminée. Les échantillons étaient déplacés avec un mouvement uniforme, et le rombre de tirs reçus par chaque élément de la surface était déterminé par les vitesses de déplacement des tables et la fréquence de répétition du laser.

Trois types d'échantillons ont été utilisés pour tester l'efficacité du procédé de décontamination. Des échantillons en acier inoxydable (316L) et en inconel provenant d'un générateur de vapeur d'un réacteur à eau pressurisée (REP), et d'autres en aluminium issus d'une installation de retraitement de combustible. La couche d'oxydes créés dans le générateur de vapeur est très épaisse $(>10 \mu \mathrm{m})$, car ils sont formés dans l'eau sous forte pression (170bar) et haute température $\left(300^{\circ} \mathrm{C}\right)$. Le principal radioélément est le Cobalt ${ }^{60}$. Les échantillons d'aluminium sont recouverts par une couche d'oxyde naturelle formée à l'air dans des conditions normales de pression et de température. Les principaux radioéléments sont $\mathrm{Cs}^{137}$, Eu ${ }^{154,155}$ et $\mathrm{Sb}^{125}$. Les mesures d'activité ont été réalisées par spectroscopie des rayons gamma, à l'aide d'un détecteur au germanium.

\section{RESULTATS ET INTERPRETATIONS}

$A_{i}$ et $A_{\Gamma}$ sont respectivement les activités initiales et résiduelles des échantillons. La figure 1 montre le pourcentage d'activité enlevée $\left(100\left(\mathrm{~A}_{\mathrm{i}}-\mathrm{A}_{\mathrm{i}}\right) / \mathrm{A}_{\mathrm{i}}\right)$ sur les échantillons d'acier en fonction du nombre de tirs laser et pour différentes fluences. L'activité initiale de ces échantillons était d'environ $30000 \mathrm{~Bq} / \mathrm{cm}^{2}$. Une fluence de $0,5 \mathrm{~J} / \mathrm{cm}^{2}$ est trop faible pour initier un processus d'ablation de la couche d'oxyde, et la faible réduction d'activité observée est due à l'enlèvement des poussières radioactives non fixées à la surface. Pour les fluences supérieures à $1 \mathrm{~J} / \mathrm{cm}^{2}$, un plasma d'ablation est clairement visible à la surface du matériau irradié. Toutes les courbes ont sensiblement la même évolution lorsque le nombre de tirs augmente, sauf le début de la courbe à $2,5 \mathrm{~J} / \mathrm{cm}^{2}$ qui est légèrement différent. En effet, le nombre limité d'échantillons nous a contraint à réutiliser, pour les essais à $2,5 \mathrm{~J} / \mathrm{cm}^{2}$, l'échantillon précédemment irradié à $0,5 \mathrm{~J} / \mathrm{cm}^{2}$ et qui ne comportait donc plus de contamination labile. La décontamination par laser est très efficace lors de la première centaine de tirs $\left(50 \%\right.$ à $\left.2 \mathrm{~J} / \mathrm{cm}^{2}\right)$, puis la vitesse de décontamination décroît. Après 500 tirs, il devient très difficile d'enlever les oxydes encore présents sur la surface. Enfin, des pourcentages de décontamination compris entre $80 \%$ et $97 \%\left(2,5 \mathrm{~J} / \mathrm{cm}^{2}\right)$ sont obtenus après 1000 tirs. 
La figure 2 montre les résultats obtenus avec 2 échantillons en inconel irradiés à $1,4 \mathrm{~J} / \mathrm{cm}^{2}$ et $3 \mathrm{~J} / \mathrm{cm}^{2}$. Leur activité initiale était de $8000 \mathrm{~Bq} / \mathrm{cm}^{2}$. Les courbes sont similaires à celles obtenues avec l'acier inoxydable, mais les pourcentages de décontamination mesurés sont plus faibles.

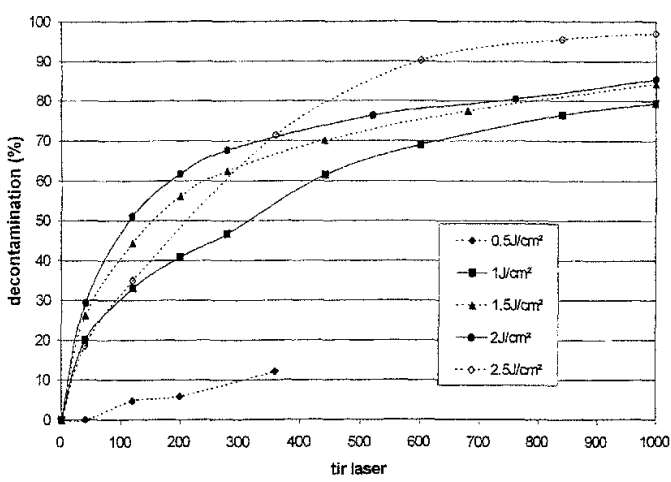

Figure 1. pourcentage de décontamination en fonction du nombre de tirs laser pour les échantillons en acier.

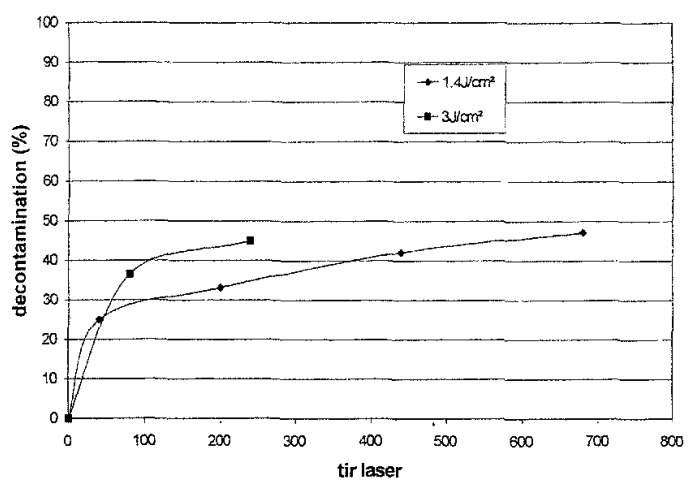

Figure 2. pourcentage de décontamination en fonction du nombre de tirs laser pour les échantillons en inconel.

Pour ces deux matériaux, le processus de décontamination peut se diviser en trois étapes. Lors des premiers tirs, la contamination labile est facilement enlevée par l'irradiation laser, même à basse fluence. Puis, une fine épaisseur d'oxyde est ablatée par chaque tir laser. Enfin, lorsque la couche d'oxyde formée au-dessus de la surface est supprimée, les oxydes piégés dans les fissures du matériau sont pratiquement impossible à faire disparaître. Ces fissures sont créées dans les générateurs de vapeur du fait des conditions de pression et de température. Elles sont moins nombreuses et plus petites pour l'acier que pour l'inconel, dans lequel elles peuvent atteindre $150 \mu \mathrm{m}$ de profondeur. Ce dernier point peut expliquer la différence entre les résultats de décontamination obtenus entre ces deux matériaux.

La figure 3 présente les résultats obtenus lors du nettoyage des échantillons en aluminium. L'activité initiale des échantillons variait entre 2000 et $12000 \mathrm{~Bq} / \mathrm{cm}^{2}$. A faible fluence $\left(1,4 \mathrm{~J} / \mathrm{cm}^{2}\right)$, aucun plasma d'ablation n'a été observé et le pourcentage de décontamination mesuré est attribué à l'enlèvement des particules non fixées. Lorsque la fluence de traitement est supérieure au seuil d'ablation de l'oxyde d'aluminium, principalement $\mathrm{Al}_{2} \mathrm{O}_{3}$, dans lequel sont piégés les radioéléments, un plasma intense apparaît et la couche d'oxyde est enlevée après quelques tirs laser. L'oxyde ayant été formé à température ambiante et pression atmosphérique, son épaisseur est faible $(\sim 1 \mu \mathrm{m})$, et il $\mathrm{n}$ 'y a quasiment pas de pénétration de la contamination à l'intérieur de la matière. Le dispositif expérimental utilisé pour tenir les échantillons d'aluminium lors de leur traitement ne nous a malheureusement pas permis d'irradier toute leur surface, et une décontamination totale n'a donc pas pu être obtenue.

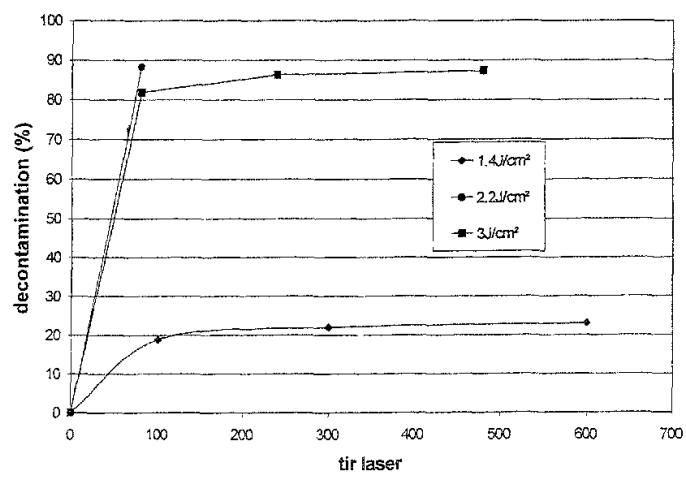

Figure 3. pourcentage de décontamination en fonction du nombre de tirs laser pour les échantillons en aluminium. 
La vitesse de décontamination du procédé laser peut être estimée à partir de la formule suivante :

$$
V=\frac{P}{F \cdot N}
$$

où $P$ est la puissance moyenne du laser à la surface de l'objet à décontaminer, $F$ la fluence d'irradiation et $\mathrm{N}$ le nombre de tirs effectué sur chaque élément de surface. Donc, avec un laser de $1 \mathrm{~kW}$, tel que le CILAS UV 635 et un faisceau de fibres de 10 mètres de long induisant une perte de puissance de $50 \%$, on peut considérer que $\mathrm{P}=500 \mathrm{~W}$. Dans le cas du générateur de vapeur, un pourcentage de décontamination de $62 \%$ est obtenu avec une vitesse $0,45 \mathrm{~m}^{2} / \mathrm{h}\left(2 \mathrm{~J} / \mathrm{cm}^{2}, 200\right.$ tirs $)$, ou de $90 \%$ avec une vitesse de $0,12 \mathrm{~m}^{2} / \mathrm{h}$ $\left(2,5 \mathrm{~J} / \mathrm{cm}^{2}, 600\right.$ tirs). Pour des contaminations moins sévères, comme celle des échantillons d'aluminium, des pourcentages de décontamination supérieurs à $90 \%$ peuvent être atteints avec une vitesse de décontamination au moins égale à $1 \mathrm{~m}^{2} / \mathrm{h}\left(2,2 \mathrm{~J} / \mathrm{cm}^{2}, 80 \mathrm{tirs}\right)$.

Pour que ce procédé soit efficace, il est important de récupérer l'ensemble des radioéléments enlevés de la surface. Lors de nos expériences, la quasi - totalité des particules ablatées a été piégée par le système de filtration. Une faible quantité d'activité a été mesurée dans la buse d'aspiration, mais aucun autre point de la boîte à gants ou du système de pompage n'a présenté de traces de contamination.

\section{CONCLUSION}

Ces expériences ont démontré l'efficacité du procédé de décontamination par laser à excimères. Pour la contamination labile ou les couches d'oxyde formées dans des conditions normales de pression et de température, quelques tirs laser sont suffisants pour effectuer une décontamination efficace de la surface. Le procédé laser permet alors d'obtenir les meilleurs rendements de nettoyage par procédé voie sèche (la décontamination électrolytique permet également d'atteindre des pourcentages supérieurs à $90 \%$, mais n'est pas adaptée au nettoyage de grandes surfaces). Pour des matériaux contaminés dans des conditions plus sévères de pression et température, quelques centaines de tirs permettent l'enlèvement de la couche d'oxyde. Cependant, les radioéléments piégés dans les fissures du matériau sont très difficiles à supprimer. Dans ce cas, le procédé laser est toujours la meilleure technique par voie sèche, mais les procédés chimiques permettent d'atteindre des facteurs de décontamination plus élevés.

Le procédé de décontamination développé autour d'un laser à excimères est une technique par voie sèche, propre et bien adapté pour le nettoyage de surfaces de grandes dimensions (vitesse de $1 \mathrm{~m}^{2} / \mathrm{h}$ pour l'aluminium). Les facteurs de décontamination obtenus sont suffisamment élevés pour utiliser ce procédé pour l'enlèvement de la plupart des contaminations rencontrées dans le milieu nucléaire.

\section{Remerciements}

Les auteurs remercient l'ANVAR et la société ONECTRA pour leur soutien à ce projet.

\section{Références}

[1] Y. Lu, M. Takia, S. Komuro, T. Shiokawa, Y. Aoyagi, 'Surface cleaning of metals by pulsed-laser irradiation in air'. Applied Physics A, 59, pp. 281-288, (1994)

[2] A. Kearns et al. 'Laser removal of oxydes from a copper substrate using Q-switched Nd:YAG radiation at $1064 \mathrm{~nm}, 532 \mathrm{~nm}$ and $226 \mathrm{~nm}$ '. Applied Surface Science 127-129, pp.773-780 (1998)

[3] M. Sentis, Ph. Delaporte, W. Marine, O. Uteza, 'Surface oxyde removal by $\mathrm{XeCl}$ laser for decontamination'. Quantum Electronics 30 (6), pp. 495-500, (2000)

[4] F. Dausinger, J Shen. 'Energy coupling efficiency in laser surface treatement', ISIJ International, 33, $N^{\circ}$ 9, pp. 925-933, (1993).

[5] Y. Lu, W. Song, C. Tee, D. Chan, T. Low. 'Wavelength effects in laser cleaning process'. Japanese Journal of Applied Physics, 37, pp. 840-844, (1998)

[6] A.S. Gouillon. 'Etude de la transmission d'un faisceau laser de grande puissance moyenne par fibre optique'. Thèse de l'université d'Aix-Marseille III. Novembre 1998.

[7] A. Kabashin, P. Nikitin, W. Marine, M. Sentis, 'Electric field of a laser plasma formed by optical breakdown of air near various targets'. Quantum Electronics 28, pp. 24-28, (1998) 